\title{
ALGEBRAS OF FINITE SELF-INJECTIVE DIMENSION
}

\author{
MITSUO HOSHINO
}

(Communicated by Maurice Auslander)

\begin{abstract}
Let $A$ be an artin algebra. Then $A$ has finite self-injective dimensions on both sides if and only if every finitely generated left $A$-module has finite Gorenstein dimension.
\end{abstract}

Two decades ago, Auslander and Bridger [2] showed that a commutative noetherian local ring $A$ is a Gorenstein ring if and only if every finitely generated $A$-module has finite Gorenstein dimension. In this paper, we will develop their arguments and apply obtained results to artin algebras. We will prove the following:

Theorem. Let $A$ be an artin algebra. Then inj $\operatorname{dim}_{A} A=\operatorname{inj} \operatorname{dim} A_{A}<\infty$ if and only if every finitely generated left A-module has finite Gorenstein dimension.

In the following, we will work over a left and right noetherian ring $A$. We denote by ()$^{*}$ both $A$-dual functors. Right $A$-modules are considered as left $A^{\mathrm{op}}$-modules, where $A^{\mathrm{op}}$ stands for the opposite ring of $A$, and notations for left $A$-modules are used also for left $A^{\mathrm{op}}$-modules. Denote by $\bmod A$ the category of all finitely generated left $A$-modules. For $n>0$, a module $M \in$ $\bmod A$ is said to be an $n$th syzygy if there is an exact sequence $0 \rightarrow M \rightarrow$ $P_{n-1} \rightarrow \cdots \rightarrow P_{0}$ in $\bmod A$ with the $P_{i}$ projective. Let $\mathrm{syz}^{n} A$ be the class of all $M \in \bmod A$ that are $n$th syzygies, and, for the sake of convenience, put $\operatorname{syz}^{0} A=\bmod A$ (i.e., every $M \in \bmod A$ is considered as a 0 th syzygy of itself). Also, a module $M \in \bmod A$ is said to have reduced grade at least $n$, written reduced grade $M \geqq n$, if $\operatorname{Ext}_{A}^{i}(M, A)=0$ for $1 \leqq i<n$. Note that reduced grade $M \geqq 1$ for all $M \in \bmod A$. Finally, a module $M \in \bmod A$ is said to have Gorenstein dimension zero, written G-dim $M=0$, if it is reflexive and reduced grade $M=$ reduced grade $M^{*}=\infty$. Then, for $n>0, M$ is said to have Gorenstein dimension at most $n$, written $\mathrm{G}-\operatorname{dim} M \leqq n$, if there is an exact sequence $0 \rightarrow M_{n} \rightarrow \cdots \rightarrow M_{0} \rightarrow M \rightarrow 0$ in $\bmod A$ with G-dim $M_{i}=0$ for $0 \leqq i \leqq n$. Note that $\mathrm{G}-\operatorname{dim} M<\infty$ implies $\operatorname{Ext}_{A}^{i}(M, A)=0$ for $i>$ G-dim $M$ (see Auslander and Bridger [2] for details).

Received by the editors March 12, 1990.

1980 Mathematics Subject Classification (1985 Revision). Primary 16A46, 16A60, 16A62; Secondary $16 \mathrm{~A} 33,16 \mathrm{~A} 35$. 


\section{PROOF OF THEOREM}

We divide the proof into several steps.

Lemma 1. Let $M \in \bmod A$ have infinite reduced grade. Then G-dim $M<\infty$ implies G-dim $M=0$.

Proof. See Auslander and Bridger [2, p. 95].

Lemma 2. Let $P_{1} \stackrel{f}{\longrightarrow} P_{0} \rightarrow M \rightarrow 0$ be an exact sequence in $\bmod A$ with the $P_{i}$ projective. Put $N=\operatorname{Cok} f^{*}$. Then $M$ is reflexive if and only if reduced grade $N \geqq 3$.

Proof. See Auslander [1, Proposition 6.3].

Lemma 3. Let $M \in \bmod A$ have reduced grade $\geqq n$. Let $P_{n} \stackrel{f}{\longrightarrow} P_{n-1} \rightarrow \cdots \rightarrow$ $P_{0} \rightarrow M \rightarrow 0$ be an exact sequence in $\bmod A$ with the $P_{i}$ projective. Then $N=\operatorname{Cok} f^{*}$ has reduced grade $\geqq n$.

Proof. We have an exact sequence $P_{0}^{*} \rightarrow \cdots \rightarrow P_{n-1}^{*} \rightarrow P_{n}^{*} \rightarrow N \rightarrow 0$ in $\bmod A^{\mathrm{op}}$ with the $P_{i}^{*}$ projective. Since each $P_{i}$ is reflexive, if ()$^{*}$ is applied, the induced sequence $0 \rightarrow N^{*} \rightarrow P_{n}^{* *} \rightarrow P_{n-1}^{* *} \rightarrow \cdots \rightarrow P_{0}^{* *}$ is exact, which implies reduced grade $N \geqq n$.

Lemma 4. For any $n \geqq 1$, the following are equivalent:

(1) Every $M \in \bmod A$ of reduced grade $\geqq n$ is reflexive.

(2) Every $N \in \bmod A^{\text {op }}$ of reduced grade $\geqq n$ has infinite reduced grade.

Proof. (1) $\Rightarrow(2)$. Let $N \in \bmod A^{\text {op }}$ have reduced grade $\geqq n$. Let $P_{n} \stackrel{f}{\longrightarrow}$ $P_{n-1} \rightarrow \cdots \rightarrow P_{0} \rightarrow N \rightarrow 0$ be an exact sequence in $\bmod A^{\text {op }}$ with the $P_{i}$ projective. Put $L=\operatorname{Cok} f$ and $M=\operatorname{Cok} f^{*}$. Then by Lemma 3 reduced grade $M$ $\geqq n$ and $M$ is reflexive. Thus by $\operatorname{Lemma} 2 \operatorname{Ext}_{A}^{n}(N, A) \simeq \operatorname{Ext}_{A}^{1}(L, A)=0$ and we get reduced grade $N \geqq n+1$. Now, by applying this argument to syzygies of $N$, we conclude that $N$ has infinite reduced grade.

$(2) \Rightarrow(1)$. Let $M \in \bmod A$ have reduced grade $\geqq n$. Let $P_{n} \stackrel{f_{n}}{\longrightarrow} P_{n-1} \rightarrow$ $\ldots \stackrel{f_{1}}{\longrightarrow} P_{0} \rightarrow M \rightarrow 0$ be an exact sequence in $\bmod A$ with the $P_{i}$ projective. Put $L=\operatorname{Cok} f_{1}^{*}$ and $N=\operatorname{Cok} f_{n}^{*}$. Note that $L$ is an $(n-1)$ th syzygy of $N$. Since by Lemma 3 reduced grade $N \geqq n$, we have reduced grade $N=\infty$ and by Lemma $2, M$ is reflexive.

Lemma 5. For any $n \geqq 0$, the following are equivalent:

(1) $\operatorname{inj} \operatorname{dim}_{A} A \leqq n$ and every $M \in \bmod A$ of infinite reduced grade is reflexive.

(2) G-dim $M=0$ for all $M \in \operatorname{syz}^{n} A$. 
Proof. (1) $\Rightarrow(2)$. Let $M \in \operatorname{syz}^{n} A$. Then reduced grade $M=\infty$ and $M$ is reflexive. Let $P_{n+1} \stackrel{f}{\rightarrow} P_{n} \rightarrow \cdots \rightarrow P_{0} \rightarrow M \rightarrow 0$ be an exact sequence in $\bmod A$ with the $P_{i}$ projective. Put $N=\operatorname{Cok} f^{*}$. Note that $M^{*}$ is an $(n+2)$ th syzygy of $N$. Since by Lemmas 3 and 4 reduced grade $N=\infty$, we get reduced grade $M^{*}=\infty$. Thus G-dim $M=0$.

(2) $\Rightarrow(1)$. The first assertion is obvious. Since G-dim $M \leqq n$ for all $M \in \bmod A$, the last assertion follows from Lemma 1 .

Lemma 6. Suppose inj $\operatorname{dim} A_{A}<\infty$. Then G-dim $M=0$ for all $M \in \bmod A$ of infinite reduced grade.

Proof. Let $M \in \bmod A$ have infinite reduced grade. Since $M^{*} \in \operatorname{syz}^{n} A^{\text {op }}$ for all $n \geqq 0, \operatorname{inj} \operatorname{dim} A_{A}<\infty$ implies reduced grade $M^{*}=\infty$. Finally, by Lemma 4, inj $\operatorname{dim} A_{A}<\infty$ implies that $M$ is reflexive.

Lemma 7. Suppose $\operatorname{inj} \operatorname{dim}_{A} A \leqq n$. Then $\operatorname{inj} \operatorname{dim} M \leqq n$ for all $M \in \bmod A$ of finite projective dimension.

Proof. Note that for an exact sequence $0 \rightarrow M_{3} \rightarrow M_{2} \rightarrow M_{1} \rightarrow 0$ we have inj $\operatorname{dim} M_{1} \leqq \max \left\{\operatorname{inj} \operatorname{dim} M_{2}, \operatorname{inj} \operatorname{dim} M_{3}\right\}$. By induction on proj $\operatorname{dim} M<$ $\infty$, the assertion follows.

Lemma 8. Let $M \in \bmod A$ be reflexive with reduced grade $M^{*}=\infty$. Then inj $\operatorname{dim} M<\infty$ implies that $M$ is projective.

Proof. We have an exact sequence $0 \rightarrow M \stackrel{f}{\longrightarrow} P_{0} \rightarrow P_{1} \rightarrow \cdots$ in $\bmod A$ with the $P_{i}$ projective. Since $\operatorname{inj} \operatorname{dim} M<\infty$ implies $f$ splitting, the assertion follows.

Lemma 9. Let $n \geqq 0$. Suppose $\mathrm{G}-\operatorname{dim} L=0$ for all $L \in \mathrm{syz}^{n} A$. Then, for any $M \in \bmod A$, the following are equivalent:

(1) $\operatorname{proj} \operatorname{dim} M<\infty$.

(2) $\operatorname{proj} \operatorname{dim} M \leqq n$.

(3) $\operatorname{inj} \operatorname{dim} M<\infty$.

(4) $\operatorname{inj} \operatorname{dim} M \leqq n$.

Proof. By Lemma 5 we have inj $\operatorname{dim}_{A} A \leqq n$. Thus by Lemma 7 (1) $\Rightarrow(4)$. It only remains to prove $(3) \Rightarrow(2)$. Suppose $\operatorname{inj} \operatorname{dim} M<\infty$. Let $L$ be an $n$th syzygy of $M$. Then $\operatorname{inj} \operatorname{dim} L<\infty$. Since G-dim $L=0$, by Lemma $8 L$ is projective; namely, $\operatorname{proj} \operatorname{dim} M \leqq n$.

Lemma 10. Suppose there is some $E \in \bmod A$ which is an injective cogenerator for left $A$-modules. Suppose inj $\operatorname{dim}_{A} A \leqq n$. Then the following are equivalent:

(1) $\operatorname{inj} \operatorname{dim} A_{A} \leqq n$.

(2) Every $M \in \bmod A$ of infinite reduced grade is reflexive.

(3) $\operatorname{inj} \operatorname{dim} M \leqq n$ for all $M \in \bmod A$ of finite injective dimension. 
Proof. By Lemma $4,(1) \Rightarrow(2)$, and by Lemmas 5 and $9,(2) \Rightarrow(3)$.

(3) $\Rightarrow(1)$. Let $\cdots \stackrel{f_{2}}{\longrightarrow} P_{1} \stackrel{f_{1}}{\longrightarrow} P_{0} \rightarrow E \rightarrow 0$ be an exact sequence in $\bmod A$ with the $P_{i}$ projective. Put $M_{i}=\operatorname{Im} f_{i}$ for $i \geqq 1$. Note that $\operatorname{inj} \operatorname{dim} M_{i}<\infty$ thus $\operatorname{inj} \operatorname{dim} M_{i} \leqq n$ for all $i \geqq 1$. Hence $\operatorname{Ext}_{A}^{n+1}\left(E, M_{n+1}\right)=0$, which implies proj $\operatorname{dim} E \leqq n$. The assertion now follows from Cartan and Eilenberg [3, Chapter VI, Proposition 5.3].

Proof of theorem. "If" part. There is some $n \geqq 0$ such that G-dim $M \leqq n$ for all simple modules $M \in \bmod A$. Then $\operatorname{inj} \operatorname{dim}_{A} A \leqq n$. Thus, by Lemmas 1 and 10, $\operatorname{inj} \operatorname{dim} A_{A} \leqq n$ and by Zaks [4, Lemma A], $\operatorname{inj} \operatorname{dim}_{A} A=\operatorname{inj} \operatorname{dim} A_{A} \leqq$ $n$.

"Only if" part. Suppose $\operatorname{inj} \operatorname{dim}_{A} A=\operatorname{inj} \operatorname{dim} A_{A} \leqq n$. Then by Lemma 6 , G-dim $M=0$ for all $M \in \operatorname{syz}^{n} A$.

\section{REFERENCES}

1. M. Auslander, Coherent functors, Proc. Conf. Cat. Algebra, Springer, Berlin, 1966, pp. 189-231.

2. M. Auslander and M. Bridger, Stable module theory, Mem. Amer Math. Soc., no. 94, Amer. Math. Soc., Providence, RI, 1969.

3. H. Cartan and S. Eilenberg, Homological algebra, Princeton Univ. Press, Princeton, 1956.

4. A. Zaks, Injective dimension of semiprimary rings, J. Algebra 13 (1969), 73-86.

Institute of Mathematics, University of Tsukuba, Ibaraki 305, Japan 